\title{
Has Chinese Stock Market Become Efficient? Evidence from a New Approach
}

\author{
Max Chen ${ }^{1}$ and Yongmiao Hong ${ }^{2}$ \\ 1 Peking University, Beijing, 100871, PR China, \\ maxchen@gsm.pku.edu.cn \\ 2 Cornell University, 424 Uris Hall, Ithaca, NY 14850, U.S.A. \\ yh20@cornell.edu
}

\begin{abstract}
Using a new statistical procedure suitable to test efficient market hypothesis in presence of volatility clustering, we find significant evidence against the weak form of efficient market hypothesis for both Shanghai and Shenzhen stock markets, although they have become more efficient at the later stage. We also find that Share A markets are more efficient than Share B markets, but there is no clear evidence on which stock market, Shanghai or Shenzhen, is more efficient. These findings are robust to volatility clustering, a key feature of high-frequency financial time series. They have important implications on predictability of stock returns and on efficacy of capital asset pricing and allocation in Chinese economy.
\end{abstract}

\section{Chinese Stock Market: An Emerging Global Market}

With In this section, we briefly describe the development of Chinese stock market, which will be helpful for readers to understand our empirical findings. Thanks to the gradual but continuous and massive economic reform initiated in 1978, Chinese economy has been growing rapidly and steadily for more than two decades, with an average of annual GDP growth rate of around $9 \%$. The reforms in restructuring state-own enterprises and pricing commodity markets have recorded remarkable success (see [1], [2] and [3]). Although still different from a competitive market economy, Chinese economy has been growing out of plan and become market-driven in most sectors.

Compared to other aspects of Chinese economic reform, the development of Chinese financial market has lagged behind. The stock markets were introduced in late 1990, 13 years after the economic reform started in 1978. Although Chinese state-owned enterprises did improve productivity in 1980s, non-performing loans to state-owned enterprises by Chinese commercial banks had been accumulating. As a consequence, commercial banks are unwilling and unable to provide further loans to poor-performing state-owned enterprises. On the other hand, private savings in Chinese banking sector have been increasing, with an annual saving rate between $30 \%$ to $40 \%$ over the last two decades. Chinese government had to find a solution to this dilemma, and the setup of a stock market was apparently a natural choice. There are two stock exchanges markets in China. 
One is located in Shenzhen, a southern city next to Hong Kong and one of the five special economic zones in China where market mechanisms were first introduced in China. The other stock exchange is located in Shanghai, the largest industrial and financial center in China. Both Shenzhen and Shanghai stock exchanges are regulated by the official China Securities Regulatory Commission (CSRC), which was set up in 1992, and has been responsible for regulating and monitoring Chinese stock market. CSRC offers two types of stocks - Share A and Share B, for domestic and foreign investors respectively. Share A markets, traded in local (i.e., Chinese) currency, are designed for domestic investors, and Share B markets, introduced in 1992 and traded in major foreign currencies, are designed for foreign investors. The introduction of Share B markets aims at attracting foreign capitals, but Share A and B markets were segmented to avoid adverse impact of financial turmoil from international financial markets. Effort has been made to merge these two markets. Starting from 02/2001, CSRC allows domestic investors holding foreign currencies to invest in Share B markets, and starting from 12/2002, qualified foreign institutional investors - mutual fund management institutions, insurance companies, securities companies, commercial and investment banks are allowed to invest in Share A markets.

Although starting relatively late, Chinese stock market, equipped with most advanced hardware facilities in the world, has developed rather rapidly. Its information delivering system, via its trading network, Internet and Reuters terminals, provides Chinese market updates in a timely manner to all domestic stock trading branches across China and to 150 counties and regions over the world. A great deal of effort has also been devoted to improving market structures, legal systems, and institutional arrangements. The stock market has been playing an increasingly important role in Chinese economy. By the end of October 2002, 1,215 companies, the majority of them stated-own, are listed in Shanghai and Shenzhen stock exchanges markets. By the end of 2001, the total market capitalization was 4,352.2 billion yuan, or $45.37 \%$ of Chinese GDP, and the tradable capitalization was 1,446.3 billion yuan, or $15.08 \%$ of Chinese GDP.

This later ratio of capitalization is still low compared to mature stock markets, but it is 10 times more than the ratio in 1991. Chinese stock market has apparently become the largest emerging stock market in the world.

Unlike the mature stock markets in developed economies, the majority of investors in Chinese stock market are individuals, accounting for more than $99 \%$ in 2002 in terms of the number of opened accounts. Institutional investors only accounts for about $0.5 \%$ in 2002 , but they contribute about $60 \%$ of investment in Chinese stock markets. Also, compared to mature stock markets, the turnover rates and $\mathrm{P} / \mathrm{E}$ ratios in Chinese stock market have been very high, indicating over-speculative activity.

Given its potential in scale, rapid growth, increasing openness to and integration with international financial markets, it is important and interesting to investigate how Chinese stock market has been performing over the past decade. In this paper, we shall examine whether Chinese stock market has achieved the weak form of market efficiency in the sense of Fama $(1970,1991)$. On one hand, 
although Chinese government still has certain direct or indirect control of financial resources such as regulating the number of new listed companies, as well as the quota and initial prices of new listed stocks, Chinese stock market has had the most flexible market mechanism in Chinese economy. With the improved legal systems and institutional arrangements, one may expect that Chinese stock market has achieved some form of market efficiency. On the other hand, the existence of government policy intervention, insider trading, revealment of misleading information on listed companies, and some irrational behavior of Chinese individual investors might lead one to expect that Chinese stock market has not been efficient. Market (in)efficiency has immediate interest to both domestic and foreign investors. For example, the predictability of stock returns is closely related to the market timing ability of mutual fund managers for active fund management. More importantly, market (in)efficiency has far-reaching implications for the efficacy of capital asset allocation and pricing. The study of EMH can also shed some light on the development of other emerging financial markets, particularly those of transitional economies (e.g., Russia and Eastern Europe). With the availability of a decade of daily observations, we now have a sufficiently large sample to provide reliable statistical inference on EMH for Chinese stock market.

\section{Efficient Market Hypothesis}

We now discuss the definition of the weak form of market efficiency, its implication and a suitable and powerful test.

\subsection{Weak Form of Efficient Market Hypothesis}

Let $P_{t}$ be a stock price at time $t$. We define the stock return at time $t$ in terms of the relative percentage change in the stock price $P_{t}$ from time $t-1$ to time $t$ :

$$
Y_{t}=100 \ln \left(P_{t} / P_{t-1}\right)
$$

Let $I_{t-1}$ denotes the collection of stock returns available at time $t-1$; i.e., $I_{t-1}=\left\{Y_{t-1}, Y_{t-2}, \ldots\right\}$. In exploring the dynamics of how stock return $Y_{t}$ changes over time, an important and interesting hypothesis is the weak form of EMH, which can be formally stated as

$$
\mathbf{H}_{0}: \quad E\left(Y_{t} \mid I_{t-1}\right)=E\left(Y_{t}\right) \text { almost surely (a.s.). }
$$

intuitively, the unconditional mean $E\left(Y_{t}\right)$ is the long-run average stock return, and the conditional mean $E\left(Y_{t} \mid I_{t-1}\right)$ is the best one-step-ahead stock return one can expect to obtain by fully and efficiently utilizing $I_{t-1}$, the information on the entire past history of stock returns. When $\mathbf{H}_{0}$ in (2) holds, no trading strategy can beat the market systematically by earning an extra return higher than the market average over the long run while bearing the same risk. Of course, investors may still be able to beat the market in the short-run by sheer luck. 
When $I_{t-1}$ is extended to include other information, we could define the semistrong form or strong form of market efficiency. See [4], [5] and [6] for more discussion. We emphasize that EMH in form of (2) can be derived from the stochastic Euler equation associated with an intertemporal utility maximization of a representative investor subject to a budget constraint (e.g., [7]).

\subsection{A Generalized Spectral Derivative Approach}

Hong and Lee [8] propose a specification test for the adequacy of a time series conditional mean model with estimated parameters. It is based on the generalized spectrum proposed in [9], which is an analytic tool for nonlinear time series, just as power spectrum is an analytic tool for linear time series (cf. [10], [11]).

Suppose $\left\{Y_{t}\right\}$ is a strictly stationary process with marginal characteristic function $\varphi(u) \equiv E\left(e^{i u Y_{t}}\right)$ and pairwise joint characteristic function $\varphi_{j}(u, v) \equiv$ $E\left(e^{i u Y_{t}+i v Y_{t-|j|}}\right)$, where $i \equiv \sqrt{-1}, u, v \in(-\infty, \infty)$ and $j=0, \pm 1, \cdots$. The basic idea of the generalized spectrum is to first transform the data via an exponential function

$$
Y_{t} \longrightarrow \exp \left(i u Y_{t}\right)
$$

and then consider the spectrum of the transformed series $\left\{e^{i u Y_{t}}\right\}$ :

$$
f(\omega, u, v) \equiv \frac{1}{2 \pi} \sum_{j=-\infty}^{\infty} \sigma_{j}(u, v) e^{-i j \omega}, \quad \omega \in[-\pi, \pi]
$$

where $\omega$ is the frequency, $\sigma_{j}(u, v)$ is the autocovariance function of the transformed series:

$$
\sigma_{j}(u, v) \equiv \operatorname{cov}\left(e^{i u Y_{t}}, e^{i v Y_{t-|j|}}\right), \quad j=0, \pm 1, \cdots
$$

The function $f(\omega, u, v)$ can capture any type of pairwise serial dependence in $\left\{Y_{t}\right\}$, i.e., dependence between $Y_{t}$ and $Y_{t-j}$ for any nonzero lag $j$, including the dependent processes with zero autocorrelation. The generalized spectrum $f(\omega, u, v)$ itself is not suitable for testing $\mathrm{EMH}$, because it can capture serial dependence not only in mean but also in higher order conditional moments. For example, the generalized spectrum $f(\omega, u, v)$ can capture the ARCH process, which is an m.d.s.

The resulting test statistic in [8] is

$$
M_{1}(p) \equiv\left[\sum_{j=1}^{T-1} k^{2}(j / p)(T-j) \int\left|\hat{\sigma}_{j}^{(1,0)}(0, v)\right|^{2} d W(v)-\hat{C}_{1}(p)\right] / \sqrt{\hat{D}_{1}(p)}
$$

Where, $p \equiv p(T)$ is a smoothing parameter called bandwidth, and $k(\cdot)$ is a symmetric kernel function that assigns a weight to each lag $j$. Examples of $k(\cdot)$ include the Bartlett kernel and the Parzen kernel. And $W: \mathbf{R} \longrightarrow \mathbf{R}^{+}$is a nondecreasing function that weights set about zero equally,

$$
\hat{C}_{1}(p)=\sum_{j=1}^{T-1} k^{2}(j / p) \frac{1}{T-j} \sum_{t=j+1}^{T-1}\left(Y_{t}-\bar{Y}\right)^{2} \int\left|\hat{\psi}_{t-j}(v)\right|^{2} d W(v)
$$




$$
\begin{gathered}
\hat{D}_{1}(p)=2 \sum_{j=1}^{T-2} \sum_{l=1}^{T-2} k^{2}(j / p) k^{2}(l / p) \times \\
\iint\left|\frac{1}{T-\max (j, l)} \sum_{t=\max (j, l)+1}^{T}\left(Y_{t}-\bar{Y}\right)^{2} \hat{\psi}_{t-j}(v) \hat{\psi}_{t-l}\left(v^{\prime}\right)\right|^{2} d W(v) d W\left(v^{\prime}\right)
\end{gathered}
$$

where $\hat{\psi}_{t}(v)=e^{i v Y_{t}}-\hat{\varphi}(v)$, and $\hat{\varphi}(v)=T^{-1} \sum_{t=1}^{T} e^{i v Y_{t}}$. Under EMH, we have

$$
M_{1}(p) \rightarrow N(0,1) \text { in distribution }
$$

provided $p \equiv p(T) \rightarrow \infty$ as $T \rightarrow \infty$ and certain regularity conditions hold. ${ }^{3}$ Upper-tailed $\mathrm{N}(0,1)$ critical values (e.g., 1.645 at the $5 \%$ level) should be used.

The $M_{1}(p)$ test has other appealing features. As explained earlier, the generalized spectral derivative $f^{(0,1,0)}(\omega, 0, v)$ only focuses on checking serial dependence in conditional mean, and thus is suitable to test EMH. It will not falsely reject EMH when there exists volatility clustering and serial dependence in higher order conditional moment. On the other hand, $f^{(0,1,0)}(\omega, 0, v)$ can detect both linear and nonlinear departures from EMH. Thus, it has more power against a wider range of departures from EMH than any autocorrelation-based tests, even if the latter were applicable in the presence of conditional heteroskedasticity of unknown form. Moreover, the $M_{1}(p)$ test can check a large number of lags. This will ensure power against departures from EMH at an unknown high lag order. Usually, the use of a large number of lags might be not powerful against many practical alternatives, due to the loss of a large number of degrees of freedom. Fortunately, this is not the case with the $M_{1}(p)$ test, which discounts higher order lags via the kernel $k(\cdot)$. The downward weighting by $k(\cdot)$ ensures good power of $M_{1}(p)$ in practice because it is consistent with the stylized fact that financial markets are usually more affected by the recent past events than by the remote past events. This is one of the advantage of frequency domain analysis over time domain analysis. The latter usually gives equal weighting to each lag, which is obviously not efficient when a large number of lags are used.

\section{Data}

Historically the majority of studies on EMH have focused on the predictability of common stock returns. Likewise, we will consider eight major stock indices in both Shanghai and Shenzhen stock markets from 12/1990 to 10/2002, obtained

\footnotetext{
${ }^{3}$ In Hong and Lee (2002), the observed raw data $\left\{Y_{t}\right\}_{t=1}^{T}$ is replaced with a sample of estimated residuals $\left\{\hat{\varepsilon}_{t}\right\}_{t=1}^{T}$, where $\hat{\varepsilon}_{t}=Y_{t}-g\left(I_{t-1}, \hat{\theta}\right), g(\cdot, \cdot)$ is a conditional mean model, and $\hat{\theta}$ is a $\sqrt{T}$-consistent finite-dimensional parameter estimator. Hong and Lee (2002) show that the limit distribution of $M_{1}(p)$ does not depend on parameter estimation uncertainty (i.e., one can treat estimated parameters as if it were equal to the true parameter values). As a consequence, one can use $M_{1}(p)$ to test EMH without any modification. The EMH hypothesis involves no parameter estimation.
} 
from China Stock Market \& Accounting Research (CSMAR) Database and WISe from Shanghai Wind Information Corporation. These indices are Shanghai Composite (SHC) index, Shanghai Share A (SHA) index, Shanghai Share B (SHB) index, Shanghai 180 (SH180) index, Shenzhen Composite (SZC) index, Shenzhen Share A (SZA) index, Shenzhen Share B (SZB) index, and Shenzhen Constituent (SZCS) index. They are most representative of the overall performance of Shanghai and Shenzhen stock markets. Their starting dates are between 1990 and 1992, except for SH180, which starts from 07/01/1996. All eight indices have the same ending date, $10 / 31 / 2002$. There exists rather strong volatility clustering in both Shanghai and Shenzhen markets, and in both Share A and Share B markets. However, it appears that Share A and Share B indices have different volatility clustering patterns. Except for Share B indices, there were more variations in the early part of the sample period (corresponding to the subsample before 12/16/1996) than in the later part of the sample period (corresponding to the period after $12 / 16 / 1996)$. This was perhaps due to the implementation of a $10 \%$ band limit on daily stock price changes with a $T+1$ settlement rule. In contrast, for Share B indices, there were more variations in the later part of the sample than in the early part of the sample period. This indicates the booming of Share B markets, which might be due to the introduction of a legal regularion by Chinese government on 01/1996 to encourage foreign investment in Share B markets.

The histograms compare the unconditional distributions of stock returns with a normal distribution having the same sample mean and sample variance. All stock returns are apparently nonnormal. They all have a higher peak around zero and heavier tails than the normal distribution, implying a large excess kurtosis. There are some extreme large stock returns, both positive and negative. For Share A and Composite indices, Shanghai market has a higher average return (sample mean) than Shenzhen market for the whole sample and for the subsamples respectively, although the sample standard deviations are not always larger. For Share B indices, Shanghai market has a smaller average return and a smaller standard deviation than Shenzhen market for the whole sample and the first subsample, but it has a higher return with a smaller standard deviation than Shenzhen market for the second subsample.

For indices rather than Share B indices, the standard deviations over the whole sample and over the first subsample are larger than the standard deviations over the second subsample. For Share B indices, however, the standard deviations are larger for the second subsample than for the whole sample and for the first subsample.

There is no strong evidence for skewness except for Shanghai Composite and Share A indices, and Shenzhen Constintuent Index. For all indices, skewness over the whole sample and over the first subsample is all positive; for the second subsample, all indices have a smaller skewness and some of them have a negative skewness. All stock returns have a larger kurtosis than implied by the normal distribution over the whole sample and over the two subsamples. The kurtosis is much larger over the whole sample and over the first subsample than over 
the second subsample. Both Shanghai Composite and Share A indices have an extremely large kurtosis over the whole sample and over the first subsample. Shenzhen Constituent index also has a very large kurtosis over the first subsample. This is apparently due to one or a few extreme market movements, such as the one on $05 / 21 / 1992$, when a daily return of $105 \%$, or a $72 \%$ return in log-price difference for Shanghai Composite index is recorded.

\section{Empirical Evidence}

Because of the existence of persistent volatility clustering in Chinese stock returns, we should use a statistical procedure that is robust to conditional heteroskedasticity of unknown form in testing EMH. Except for SH180 with $c \leq 5$, all indices are firmly rejected at the $5 \%$ significance level for all $c$. The strong rejection of EMH might be due to imperfect institutional arrangement, frequent government policy intervention, and the irrational investors' behavior in the stock market at the early stage. To examine whether Chinese stock market has achieved EMH, or whether Chinese stock market has become more effiicient at the later stage, we also examine the subsamples before and after 12/16/1996, when Chinese stock market began to implement a $10 \%$ band limit on daily stock price changes. Before this date, transaction rules were changed several times by governments according to stock market conditions. Since 1997, CSRC has taken charge of both Shenzhen and Shanghai stock markets, marking the end of the experimental period and the beginning of a new development period for a more unified Chinese stock market.

From the first subsample, the results indicate the rejection of EMH for all indices except Shenzhen Share A and Shanghai 180 indices, for most choices of $c$ at the $1 \%$ level. Note that SH180 only has 115 observations before 12/16/1996, so the insingifinat result with SH180 may be due to the small sample problem.

For most choices of $c$, the values of $M_{1}\left(\hat{p}_{0}\right)$ based on the second subsample reject $\mathrm{EMH}$ for all eight indices at the $5 \%$ significance level, including Shenzhen Share A and SH180 indices. There appears some evidence that there exists departures from EMH at higher order lags (which implies a longer-run mean reverting) for the Composite and Share A indices, because the $M_{1}\left(\hat{p}_{0}\right)$ statistics become larger and significant when $c$ becomes larger.

Why has Chinese stock market not become efficient after a decade? Because this is an important issue, we are tempted to provide some speculations here. First, Chinese stock market, although one decade has passed since its setup in 1990, remains far away from a mature capitalist stock market. Unlike commodity markets in Chinese economy, where prices are basically determined by market forces, Chinese government still has direct and indirect control of financial resources, including the number of new listed companies as well as the quota and initial prices of new listed stocks. Stock prices do not fully incorporate all available public information. Investors have to respond to non-price signals. In particular, they have to speculate somewhat irregular government policy intervention, although the scope and frequency of policy intervention have been 
diminished since 1997. Second, for a capital market to function well, it is important for investors to receive high-quality information on listed companies in a timly manner. However, there have been many instances that listed companies may deliver misleading information on their financial accounting data. In addition, the existence of price limit (e.g., the $10 \%$ band limit), market segmentation, and insider trading may have slowed down information flow to investors. Of course, more careful empirical examination is needed to sort out possible sources of market inefficiency, but this is beyond the scope of the present paper.

A comparison between the two subsamples suggests that except for Shenzhen Share A and SH180 indices, the $M_{1}\left(\hat{p}_{0}\right)$ statistic is generally larger in the first subsample than in the second subsample. Given that the two subsamples have similar sample sizes for all indices except SH180, this indicates that both Shanghai (in terms of Composite, Share A and Share B indices) and Shenzhen (in terms of Composite, Constituent, and Share B indices) stock markets have become more efficient at the later stage. It appears that Chinese government's regulation of Chinese stock market has apparently been improving and working in the right direction.

A comparison of the whole and the two subsamples also reveals that for both Shanghai and Shenzhen markets, the values of $M_{1}\left(\hat{p}_{0}\right)$ are much larger for Share B indices than Share A indices. This indicates that for both Shanghai and Shenzhen stock markets, Share A markets are more efficient than Share B markets. We note that there is no clear systematic pattern on which market, Shanghai or Shenzhen, is more efficient. Both Shanghai and Shenzhen stock exchanges have been competing in building up Chinese stock market.

Why are Share B markets less efficient than Share A markets? A well-function financial market requires a high volume of transactions, which will quickly wipe out any arbitrage opportunity, thus achieving market efficiency. Share B markets have been featured with a low level of transactions for most of time over the last decade. This is so even after Chinese government opened Share B markets to domestic Chinese investors in $02 / 2001$. It may be expected with the integration between the segmented Share A and Share B markets, Share B markets will become more efficient.

The empirical findings documented here have important implications. For example, the violation of EMH implies that stock returns in Chinese stock market are predictable using the past history of stock returns. This has immediate interest to both domestic and foreign investors. In particular, it implies that it is possible to beat the market by using a suitable trading strategy. A relevant question is how one can predict stock returns in Chinese stock market. Do there exist trading strategies that have superior out-of-sample predictable ability? On the other hand, the violation of EMH has important implications on the efficacy of capital asset pricing for the listed companies in Chinese stock market. It would be interesting and important to develop an appropriate asset pricing model to examine what factors (e.g., macroeconomic factors) will help determine asset prices of listed companies in Chinese stock market. All these issues are left for subsequent research. 


\section{Conclusion}

Using a generalized spectral derivative test suitable and powerful to test EMH in presence of volatility clustering, we find significant evidence against the weak form of efficient market hypothesis for both Shanghai and Shenzhen stock markets, although there exists some evidence that they have become more efficient at the later stage. We also find that Share A markets are more efficient than Share B markets. Our findings are robust to volatility clustering of unknown form. Some speculations are given to explain the empirical findings.

We thank the seminar participants at China Center for Economic Research (CCER), Peking University, Antai School of Management, Shanghai Jiao Tong University, and School of Management, Xiamen University for comments, and the China Postdoctoral Science Foundation, the National Science Foundation of United States and School of Economics and Management, Tsinghua University for support. We thank the Research Center for Financial Mathematics and Financial Engineering, Peking University for the powerful and valuable CPU time.

\section{References}

1. Groves, T., Hong, Y., McMillan, J., Naughton, B.: Incentives in Chinese Stateowned Enterprices, Quarterly Journal of Economics. 1 (1994) 183-209.

2. Naughton, B.: Growing out of Plan, Oxford University Press: Oxford. (1994)

3. Lin, Y.F., Cai, F., Zhou, L.: China Miracle, Chinese University of Hong Kong Publisher: Hong Kong. (1996)

4. Fama, E.F.: A Review of Theory and Empirical Work, Journal of Finance. 25 (1970) 383-417.

5. Fama, E.F.: Efficient Markets II. Journal of Finance. 46 (1991) 1575-1618.

6. Campbell, C., Lo, A., MacKinlay A.C.: Econometrics of Financial Markets, Princeton University Press: Princeton, New Jersey. (1997)

7. Sargent, T., Ljungqvist, L.: Recursive Macroeconomic Theory. MIT Press: Cambridge, MA. (2002)

8. Hong, Y., Lee Y.: Generalized Spectral Tests for Conditional Mean Specification in Time Series with Conditional Heteroskedasticity of Unknown Form. Working paper, Department of Economics \& Department of Statistical Science, Cornell University. (2002)

9. Hong, Y.: Hypothesis Testing in Time Series via the Empirical Characteristic Function: A Generalized Spectral Density Approach. Journal of the American Statistical Association. (1999) 84, 1201-1220.

10. Priestley, M.B.: Spectral Analysis and Time Series. Academic press: London. (1981)

11. Hamilton, J.: Time Series Analysis, Princeton University Press: Princeton, New Jersey. (1994) 